\title{
New Efficient Estimators of Population Mean Using Non-Traditional Measures of Dispersion
}

\author{
Rajesh Kumar Gupta1, Subhash Kumar Yadav²* \\ ${ }^{1}$ Department of Statistics, Lucknow University, Lucknow, India \\ ${ }^{2}$ Department of Mathematics and Statistics (A Centre of Excellence), Dr. RML Avadh University, Faizabad, India \\ Email: rajeshgupta2004@gmail.com, *drskystats@gmail.com
}

How to cite this paper: Gupta, R.K. and Yadav, S.K. (2017) New Efficient Estimators of Population Mean Using Non-Traditional Measures of Dispersion. Open Journal of Statistics, 7, 394-404.

https://doi.org/10.4236/ojs.2017.73028

Received: January 28, 2017

Accepted: June 2, 2017

Published: June 5, 2017

Copyright $\odot 2017$ by authors and Scientific Research Publishing Inc. This work is licensed under the Creative Commons Attribution International License (CC BY 4.0).

http://creativecommons.org/licenses/by/4.0/

\begin{abstract}
One of the aims in survey sampling is to search for the estimators with highest efficiency. In the present paper, three improved estimators of population mean have been proposed using some non-traditional measures of dispersion of auxiliary variable such as Gini's mean difference, Downton's method and probability weighted moments early given by Abid [1] with a special population parameter of auxiliary variable. The large sample properties that are biased and mean squared errors of the proposed estimators have been derived up to the first order of approximation. A theoretical comparison of the proposed estimators has been made with the other existing estimators of population mean using auxiliary information. The conditions under which the proposed estimators perform better than the other existing estimators of population mean have been given. A numerical study is also carried out to see the performances of the proposed and existing estimators of population mean and verify the conditions under which proposed estimators are better than other estimators. It has been shown that the proposed estimators perform better than the existing estimators as they are having lesser mean squared error.
\end{abstract}

\section{Keywords}

Study Variable, Auxiliary Variable, Bias, Mean Squared Error, Efficiency

\section{Introduction}

Sampling is done when the population is very large and we have to get the result very soon. The population parameters are estimated by the corresponding statistics in a natural sense. As it has been mentioned that the most suitable estimator for the estimation of population parameter is the corresponding statistics so to estimate population mean the most suitable estimator is the sample mean. Although he sample mean is an unbiased estimator of population mean and it has 
reasonably large variance and our aim is to search for the estimator with minimum variance or may be biased but with minimum mean squared error. This purpose is solved through the use of auxiliary information. Auxiliary information is obtained from auxiliary variable which is highly positively or negatively correlated with main variable under study. When the auxiliary variable is positively correlated with the main variable under study, ratio type estimators are used for improved estimation of population parameters. When it is negatively with the main variable under consideration, product type estimators are used for improved estimation of population parameters. In the present manuscript, we have confined our study to positively correlated populations only and proposed three ratio type estimators for improved estimation of population mean with higher efficiencies.

Let the population under consideration consists of $N$ distinct and identifiable units and let $\left(x_{i}, y_{i}\right), i=1,2, \cdots, n$ be a two variable sample of size $n$ taken from bivariate variables $(X, Y)$ through simple random sampling without sampling scheme. Let $\bar{X}$ and $\bar{Y}$ be the population means of the auxiliary and the study variables respectively, and let $\bar{X}$ and $\bar{y}$ be the respective sample means and both are unbiased estimators of $\bar{X}$ and $\bar{Y}$ respectively. Let the correlation coefficient between the variables $X$ and $Y$ be denoted by $\rho$.

\section{Existing Estimators under Review}

As mentioned above most appropriate estimator of population mean is the sample mean $\bar{y}$ given by,

$$
t_{o}=\bar{y}=\frac{1}{n} \sum_{i=1}^{n} y_{i}
$$

The above estimator is unbiased for population mean of the study variable and its variance up to the first order of approximation is given by,

$$
V\left(t_{0}\right)=\frac{1-f}{n} S_{y}^{2}
$$

Cochran [2] proposed the following usual ratio estimator of population mean by using positively correlated auxiliary variable as,

$$
t_{R}=\bar{y} \frac{\bar{X}}{\bar{x}}
$$

This estimator is biased and the bias and mean squared error of this estimator, up to the first order of approximation respectively are given by,

$$
\begin{aligned}
& B\left(t_{R}\right)=\frac{1-f}{n} \frac{1}{\bar{X}}\left[R_{1} S_{x}^{2}-\rho S_{y} S_{x}\right] \\
& \operatorname{MSE}\left(t_{R}\right)=\frac{1-f}{n}\left[S_{y}^{2}+R_{1}^{2} S_{x}^{2}-2 R_{1} \rho S_{y} S_{x}\right],
\end{aligned}
$$

where $R_{1}=\frac{\bar{Y}}{\bar{X}}$

Many estimators of population mean have been given by various authors in 
the literature for improved estimation. The latest references can be made of Yadav [3], Yadav and Kadilar [4] [5], Yadav et al. [6] [7] [8] [9], Yadav and Mishra [10], Misra and Gupta [11] [12] and Misra et al [13]. The Table 1 below represents different estimators of population mean using auxiliary variable along with their constants, biases and their mean squared errors.

Table 1. Various estimators of population mean, bias, mean squared error and constant.

\begin{tabular}{|c|c|c|c|}
\hline Estimator & Bias & Mean Squared Error & Constant \\
\hline $\begin{array}{c}t_{1}=\frac{\bar{y}+b(\bar{X}-\bar{x})}{\bar{x}} \bar{X} \\
\text { Kadilar and Cingi [14] }\end{array}$ & $B\left(t_{1}\right)=\frac{1-f}{n} \frac{S_{x}^{2}}{\bar{Y}} R_{1}^{2}$ & $\operatorname{MSE}\left(t_{1}\right)=\frac{1-f}{n}\left[R_{1}^{2} S_{x}^{2}+S_{y}^{2}\left(1-\rho^{2}\right)\right]$ & $R_{1}=\frac{\bar{Y}}{\bar{X}}$ \\
\hline $\begin{array}{c}t_{2}=\frac{\bar{y}+b(\bar{X}-\bar{x})}{\left(\bar{x}+C_{x}\right)}\left(\bar{X}+C_{x}\right) \\
\text { Kadilar and Cingi [14] }\end{array}$ & $B\left(t_{2}\right)=\frac{1-f}{n} \frac{S_{x}^{2}}{\bar{Y}} R_{2}^{2}$ & $\operatorname{MSE}\left(t_{2}\right)=\frac{1-f}{n}\left[R_{2}^{2} S_{x}^{2}+S_{y}^{2}\left(1-\rho^{2}\right)\right]$ & $R_{2}=\frac{\bar{Y}}{\bar{X}+C_{x}}$ \\
\hline $\begin{array}{c}t_{3}=\frac{\bar{y}+b(\bar{X}-\bar{x})}{\left(\bar{x}+\beta_{2}\right)}\left(\bar{X}+\beta_{2}\right) \\
\text { Kadilar and Cingi [14] }\end{array}$ & $B\left(t_{3}\right)=\frac{1-f}{n} \frac{S_{x}^{2}}{\bar{Y}} R_{3}^{2}$ & $\operatorname{MSE}\left(t_{3}\right)=\frac{1-f}{n}\left[R_{3}^{2} S_{x}^{2}+S_{y}^{2}\left(1-\rho^{2}\right)\right]$ & $R_{3}=\frac{\bar{Y}}{\bar{X}+\beta_{2}}$ \\
\hline $\begin{aligned} t_{4}= & \frac{\bar{y}+b(\bar{X}-\bar{x})}{\left(\bar{x} \beta_{2}+C_{x}\right)}\left(\bar{X} \beta_{2}+C_{x}\right) \\
& \text { Kadilar and Cingi [14] }\end{aligned}$ & $B\left(t_{4}\right)=\frac{1-f}{n} \frac{S_{x}^{2}}{\bar{Y}} R_{4}^{2}$ & $\operatorname{MSE}\left(t_{4}\right)=\frac{1-f}{n}\left[R_{4}^{2} S_{x}^{2}+S_{y}^{2}\left(1-\rho^{2}\right)\right]$ & $R_{4}=\frac{\bar{Y} \beta_{2}}{\bar{X} \beta_{2}+C_{x}}$ \\
\hline $\begin{aligned} t_{5}= & \frac{\bar{y}+b(\bar{X}-\bar{x})}{\left(\bar{x} C_{x}+\beta_{2}\right)}\left(\bar{X} C_{x}+\beta_{2}\right) \\
& \text { Kadilar and Cingi [14] }\end{aligned}$ & $B\left(t_{5}\right)=\frac{1-f}{n} \frac{S_{x}^{2}}{\bar{Y}} R_{5}^{2}$ & $\operatorname{MSE}\left(t_{5}\right)=\frac{1-f}{n}\left[R_{5}^{2} S_{x}^{2}+S_{y}^{2}\left(1-\rho^{2}\right)\right]$ & $R_{5}=\frac{\bar{Y} C_{x}}{\bar{X} C_{x}+\beta_{2}}$ \\
\hline $\begin{array}{c}t_{6}=\frac{\bar{y}+b(\bar{X}-\bar{x})}{(\bar{x}+\rho)}(\bar{X}+\rho) \\
\text { Kadilar and Cingi [15] }\end{array}$ & $B\left(t_{6}\right)=\frac{1-f}{n} \frac{S_{x}^{2}}{\bar{Y}} R_{6}^{2}$ & $\operatorname{MSE}\left(t_{6}\right)=\frac{1-f}{n}\left[R_{6}^{2} S_{x}^{2}+S_{y}^{2}\left(1-\rho^{2}\right)\right]$ & $R_{6}=\frac{\bar{Y}}{\bar{X}+\rho}$ \\
\hline $\begin{array}{c}t_{7}=\frac{\bar{y}+b(\bar{X}-\bar{x})}{\left(\bar{x} C_{x}+\rho\right)}\left(\bar{X} C_{x}+\rho\right) \\
\quad \text { Kadilar and Cingi [15] }\end{array}$ & $B\left(t_{7}\right)=\frac{1-f}{n} \frac{S_{x}^{2}}{\bar{Y}} R_{7}^{2}$ & $\operatorname{MSE}\left(t_{7}\right)=\frac{1-f}{n}\left[R_{7}^{2} S_{x}^{2}+S_{y}^{2}\left(1-\rho^{2}\right)\right]$ & $R_{\gamma}=\frac{\bar{Y} C_{x}}{\bar{X} C_{x}+\rho}$ \\
\hline $\begin{array}{c}t_{8}=\frac{\bar{y}+b(\bar{X}-\bar{x})}{\left(\bar{x} \rho+C_{x}\right)}\left(\bar{X} \rho+C_{x}\right) \\
\quad \text { Kadilar and Cingi [15] }\end{array}$ & $B\left(t_{8}\right)=\frac{1-f}{n} \frac{S_{x}^{2}}{\bar{Y}} R_{8}^{2}$ & $\operatorname{MSE}\left(t_{\mathrm{g}}\right)=\frac{1-f}{n}\left[R_{8}^{2} S_{x}^{2}+S_{y}^{2}\left(1-\rho^{2}\right)\right]$ & $R_{8}=\frac{\bar{Y} \rho}{\bar{X} \rho+C_{x}}$ \\
\hline $\begin{array}{c}t_{9}=\frac{\bar{y}+b(\bar{X}-\bar{x})}{\left(\bar{x} \beta_{2}+\rho\right)}\left(\bar{X} \beta_{2}+\rho\right) \\
\text { Kadilar and Cingi [15] }\end{array}$ & $B\left(t_{9}\right)=\frac{1-f}{n} \frac{S_{x}^{2}}{\bar{Y}} R_{9}^{2}$ & $\operatorname{MSE}\left(t_{9}\right)=\frac{1-f}{n}\left[R_{9}^{2} S_{x}^{2}+S_{y}^{2}\left(1-\rho^{2}\right)\right]$ & $R_{9}=\frac{\bar{Y} \beta_{2}}{\bar{X} \beta_{2}+\rho}$ \\
\hline $\begin{array}{c}t_{10}=\frac{\bar{y}+b(\bar{X}-\bar{x})}{\left(\bar{x} \rho+\beta_{2}\right)}\left(\bar{X} \rho+\beta_{2}\right) \\
\text { Kadilar and Cingi }[15]\end{array}$ & $B\left(t_{10}\right)=\frac{1-f}{n} \frac{S_{x}^{2}}{\bar{Y}} R_{10}^{2}$ & $\operatorname{MSE}\left(t_{10}\right)=\frac{1-f}{n}\left[R_{10}^{2} S_{x}^{2}+S_{y}^{2}\left(1-\rho^{2}\right)\right]$ & $R_{10}=\frac{\bar{Y} \rho}{\bar{X} \rho+\beta_{2}}$ \\
\hline $\begin{array}{c}t_{11}=\frac{\bar{y}+b(\bar{X}-\bar{x})}{\left(\bar{x}+\beta_{1}\right)}\left(\bar{X}+\beta_{1}\right) \\
\text { Yan and Tian [16] }\end{array}$ & $B\left(t_{11}\right)=\frac{1-f}{n} \frac{S_{x}^{2}}{\bar{Y}} R_{11}^{2}$ & $\operatorname{MSE}\left(t_{11}\right)=\frac{1-f}{n}\left[R_{11}^{2} S_{x}^{2}+S_{y}^{2}\left(1-\rho^{2}\right)\right]$ & $R_{11}=\frac{\bar{Y}}{\bar{X}+\beta_{1}}$ \\
\hline $\begin{array}{c}t_{12}=\frac{\bar{y}+b(\bar{X}-\bar{x})}{\left(\bar{x} \beta_{1}+\beta_{2}\right)}\left(\bar{X} \beta_{1}+\beta_{2}\right) \\
\text { Yan and Tian [16] }\end{array}$ & $B\left(t_{12}\right)=\frac{1-f}{n} \frac{S_{x}^{2}}{\bar{Y}} R_{12}^{2}$ & $\operatorname{MSE}\left(t_{12}\right)=\frac{1-f}{n}\left[R_{12}^{2} S_{x}^{2}+S_{y}^{2}\left(1-\rho^{2}\right)\right]$ & $R_{12}=\frac{\bar{Y} \beta_{1}}{\bar{X} \beta_{1}+\beta_{2}}$ \\
\hline
\end{tabular}




\section{Continued}

$$
t_{13}=\frac{\bar{y}+b(\bar{X}-\bar{x})}{\left(\bar{x}+M_{d}\right)}\left(\bar{X}+M_{d}\right)
$$

Subramani and Kumarpandiyan [17]

$$
t_{14}=\frac{\bar{y}+b(\bar{X}-\bar{x})}{\left(C_{x} \bar{x}+M_{d}\right)}\left(C_{x} \bar{X}+M_{d}\right)
$$

Subramani and Kumarpandiyan [17]

$$
t_{15}=\frac{\bar{y}+b(\bar{X}-\bar{x})}{\left(\bar{x} \beta_{1}+M_{d}\right)}\left(\bar{X} \beta_{1}+M_{d}\right)
$$

Subramani and Kumarpandiyan [17]

$$
t_{16}=\frac{\bar{y}+b(\bar{X}-\bar{x})}{\left(\bar{x} \beta_{2}+M_{d}\right)}\left(\bar{X} \beta_{2}+M_{d}\right)
$$

Subramani and Kumarpandiyan [17]

$$
\begin{aligned}
& t_{17}=\frac{\bar{y}+b(\bar{X}-\bar{x})}{\left(\bar{x} \beta_{1}+Q D\right)}\left(\bar{X} \beta_{1}+Q D\right) \\
& \text { Jeelani et al. [18] } \\
& t_{18}=\frac{\bar{y}+b(\bar{X}-\bar{x})}{(\bar{x}+G)}(\bar{X}+G) \\
& \text { Abid et al. [1] } \\
& t_{19}=\frac{\bar{y}+b(\bar{X}-\bar{x})}{(\bar{x} \rho+G)}(\bar{X} \rho+G) \\
& \text { Abid et al. [1] } \\
& t_{20}=\frac{\bar{y}+b(\bar{X}-\bar{x})}{\left(\bar{x} C_{x}+G\right)}\left(\bar{X} C_{x}+G\right) \\
& \text { Abid et al. [1] } \\
& t_{21}=\frac{\bar{y}+b(\bar{X}-\bar{x})}{(\bar{x}+D)}(\bar{X}+D) \\
& \text { Abid et al. [1] } \\
& t_{22}=\frac{\bar{y}+b(\bar{X}-\bar{x})}{(\bar{x} \rho+D)}(\bar{X} \rho+D) \\
& \text { Abid et al. [1] } \\
& t_{23}=\frac{\bar{y}+b(\bar{X}-\bar{x})}{\left(\bar{x} C_{x}+D\right)}\left(\bar{X} C_{x}+D\right) \\
& \text { Abid et al. [1] } \\
& t_{24}=\frac{\bar{y}+b(\bar{X}-\bar{x})}{\left(\bar{x}+S_{p w}\right)}\left(\bar{X}+S_{p w}\right) \\
& \text { Abid et al. [1] } \\
& t_{25}=\frac{\bar{y}+b(\bar{X}-\bar{x})}{\left(\bar{x} \rho+S_{p w}\right)}\left(\bar{X} \rho+S_{p w}\right) \\
& \text { Abid et al. [1] } \\
& t_{26}=\frac{\bar{y}+b(\bar{X}-\bar{x})}{\left(\bar{x} C_{x}+S_{p w}\right)}\left(\bar{X} C_{x}+S_{p w}\right) \\
& \text { Abid et al. [1] } \\
& R_{21}=\frac{\bar{Y}}{\bar{X}+D} \\
& R_{22}=\frac{\bar{Y} \rho}{X \rho+D}
\end{aligned}
$$

$B\left(t_{13}\right)=\frac{1-f}{n} \frac{S_{x}^{2}}{\bar{Y}} R_{13}^{2}$

$\operatorname{MSE}\left(t_{13}\right)=\frac{1-f}{n}\left[R_{13}^{2} S_{x}^{2}+S_{y}^{2}\left(1-\rho^{2}\right)\right]$

$R_{13}=\frac{\bar{Y}}{\bar{X}+M_{d}}$

$B\left(t_{14}\right)=\frac{1-f}{n} \frac{S_{x}^{2}}{\bar{Y}} R_{14}^{2}$

$\operatorname{MSE}\left(t_{14}\right)=\frac{1-f}{n}\left[R_{14}^{2} S_{x}^{2}+S_{y}^{2}\left(1-\rho^{2}\right)\right]$

$R_{14}=\frac{\bar{Y} C_{x}}{\bar{X} C_{x}+M_{d}}$

$B\left(t_{15}\right)=\frac{1-f}{n} \frac{S_{x}^{2}}{\bar{Y}} R_{15}^{2}$

$\operatorname{MSE}\left(t_{15}\right)=\frac{1-f}{n}\left[R_{15}^{2} S_{x}^{2}+S_{y}^{2}\left(1-\rho^{2}\right)\right]$

$$
R_{15}=\frac{\bar{Y} \beta_{1}}{\bar{X} \beta_{1}+M_{d}}
$$

$B\left(t_{16}\right)=\frac{1-f}{n} \frac{S_{x}^{2}}{\bar{Y}} R_{16}^{2}$

$\operatorname{MSE}\left(t_{16}\right)=\frac{1-f}{n}\left[R_{16}^{2} S_{x}^{2}+S_{y}^{2}\left(1-\rho^{2}\right)\right]$

$R_{16}=\frac{\bar{Y} \beta_{2}}{\bar{X} \beta_{2}+M_{d}}$ 


\section{Proposed Estimators}

Motivated by Abid et al. [1] and Subramani [19] and searching for the improved estimators, we have used a specific parameter as the ratio of correlation coefficient and coefficient of skewness of auxiliary variable along with some non-traditional parameters of auxiliary variable given by Abid et al. [1] as,

$$
\begin{aligned}
& t_{p_{1}}=\frac{\bar{y}+b(\bar{X}-\bar{x})}{(\tau \bar{x}+G)}(\tau \bar{X}+G), \\
& t_{p_{2}}=\frac{\bar{y}+b(\bar{X}-\bar{x})}{(\tau \bar{X}+D)}(\tau \bar{X}+D), \\
& t_{p_{3}}=\frac{\bar{y}+b(\bar{X}-\bar{x})}{\left(\tau \bar{X}+S_{p w}\right)}\left(\tau \bar{X}+S_{p w}\right),
\end{aligned}
$$

where, $\tau=\rho / \beta_{1}$

To study the large sample approximations, we have used the following approximations as,

$$
\bar{y}=\bar{Y}\left(1+e_{0}\right) \text { and } \bar{x}=\bar{X}\left(1+e_{1}\right)
$$

such that

$$
E\left(e_{i}\right)=0, i=0,1
$$

and

$$
E\left(e_{0}^{2}\right)=\frac{1-f}{n} C_{y}^{2}, \quad E\left(e_{1}^{2}\right)=\frac{1-f}{n} C_{x}^{2},
$$

and

$$
E\left(e_{0} e_{1}\right)=\frac{1-f}{n} C_{y x}=\frac{1-f}{n} \rho C_{y} C_{x},
$$

where $f=\frac{n}{N}, C_{y}^{2}=\frac{S_{y}^{2}}{\bar{Y}^{2}}$, and $C_{x}^{2}=\frac{S_{x}^{2}}{\bar{X}^{2}}$.

Using above approximation and up to the first order of approximations, the biases and the mean squared errors of proposed estimators are given by,

$$
\begin{aligned}
& B\left(t_{p_{j}}\right)=\frac{1-f}{n} \frac{S_{x}^{2}}{\bar{Y}} R_{p_{j}}^{2},(j=1,2,3) \\
& \operatorname{MSE}\left(t_{p_{j}}\right)=\frac{1-f}{n}\left[R_{p_{j}}^{2} S_{x}^{2}+S_{y}^{2}\left(1-\rho^{2}\right)\right],(j=1,2,3)
\end{aligned}
$$

where,

$$
R_{p_{1}}=\frac{\bar{Y} \tau}{\bar{X} \tau+G}, \quad R_{p_{2}}=\frac{\bar{Y} \tau}{\bar{X} \tau+D}, \quad R_{p_{3}}=\frac{\bar{Y} \tau}{\bar{X} \tau+S_{p w}}
$$

\section{Efficiency Comparison}

In this section, the proposed estimators have been compared theoretically with the other existing estimators of population mean in terms of theirs variances and mean squared errors under simple random sampling without replacement 
scheme.

From Equation (3) and the from the Equation (1), the proposed estimators performs better than the mean per unit estimator if,

$$
\operatorname{MSE}\left(t_{p_{j}}\right)-V(\bar{y}) \leq 0
$$

or,

$$
\left[R_{p_{j}}^{2} S_{x}^{2}-\rho^{2} S_{y}^{2}\right] \leq 0
$$

or,

$$
R_{p_{j}}^{2} \leq \frac{\rho^{2} S_{y}^{2}}{S_{x}^{2}}
$$

or,

$$
R_{p_{i}} \leq \pm \frac{\rho S_{y}}{S_{x}},(j=1,2,3)
$$

The proposed estimators $t_{p_{j}}(j=1,2,3)$ in Equation (3) are better than the ratio estimator by Cochran [2] $t_{r}$ in Equation (2) under the condition if,

$$
\operatorname{MSE}\left(t_{p_{j}}\right)-\operatorname{MSE}\left(t_{r}\right) \leq 0
$$

or,

$$
\left[\left(R_{p_{j}}^{2}-R_{1}^{2}\right) S_{x}^{2}-\rho^{2} S_{y}^{2}+2 R_{1} \rho S_{y} S_{x}\right] \leq 0
$$

or,

$$
\left(R_{p_{j}}^{2}-R_{1}^{2}\right) S_{x}^{2} \leq \rho^{2} S_{y}^{2}-2 R_{1} \rho S_{y} S_{x},(j=1,2,3)
$$

From Equation (3) and the mean squared error of the estimators given by Kadilar and Cingi [14] in Table 1, the proposed estimators perform better than the Kadilar and Cingi [14] estimators under the condition if,

$$
\operatorname{MSE}\left(t_{p_{j}}\right)-\operatorname{MSE}\left(t_{i}\right) \leq 0
$$

or,

$$
\left[R_{p_{j}}^{2} S_{x}^{2}-R_{i}^{2} S_{x}^{2}\right] \leq 0
$$

or,

$$
R_{p_{j}} \leq \pm R_{i},(j=1,2,3),(i=1,2,3,4,5)
$$

From the mean squared errors of proposed estimators and Kadilar and Cingi [15] estimators respectively in Equation (3) and in Table 1, the proposed estimators are better than the Kadilar and Cingi [15] estimators if,

$$
\operatorname{MSE}\left(t_{p_{j}}\right)-\operatorname{MSE}\left(t_{i}\right) \leq 0
$$

or,

$$
\left[R_{p_{j}}^{2} S_{x}^{2}-R_{i}^{2} S_{x}^{2}\right] \leq 0
$$


or,

$$
R_{p_{j}} \leq \pm R_{i},(j=1,2,3),(i=6,7,8,9,10)
$$

From Equation (3) and the mean squared error of the estimators given by Yan and Tian [16] in Table 1, the proposed estimators are better than Yan and Tian [16] estimators if,

$$
\operatorname{MSE}\left(t_{p_{j}}\right)-\operatorname{MSE}\left(t_{i}\right) \leq 0
$$

or,

$$
\left[R_{p_{j}}^{2} S_{x}^{2}-R_{i}^{2} S_{x}^{2}\right] \leq 0
$$

or,

$$
R_{p_{j}} \leq \pm R_{i},(j=1,2,3),(i=11,12)
$$

From Equation (3) and the mean squared errors of the estimators given by Subramani and Kumarpandiyan [17] in Table 1, the proposed estimators perform better than Subramani and Kumarpandiyan [17] estimators if,

$$
\operatorname{MSE}\left(t_{p_{j}}\right)-\operatorname{MSE}\left(t_{i}\right) \leq 0
$$

or,

$$
\left[R_{p_{j}}^{2} S_{x}^{2}-R_{i}^{2} S_{x}^{2}\right] \leq 0
$$

or,

$$
R_{p_{j}} \leq \pm R_{i},(j=1,2,3),(i=13,14,15,16)
$$

The proposed estimators are better than the estimators by Jeelani et al. [18] in Table 1 under the condition if,

$$
\operatorname{MSE}\left(t_{p_{j}}\right)-\operatorname{MSE}\left(t_{17}\right) \leq 0
$$

or,

$$
\left[R_{p_{j}}^{2} S_{x}^{2}-R_{17}^{2} S_{x}^{2}\right] \leq 0
$$

or,

$$
R_{p_{j}} \leq \pm R_{17},(j=1,2,3)
$$

From MSE of the proposed estimators in Equation (3) and the estimators given by Abid et al. [1], it is found the proposed estimators are better than Abid et al. [1] estimators if,

$$
\operatorname{MSE}\left(t_{p_{j}}\right)-\operatorname{MSE}\left(t_{i}\right) \leq 0
$$

or,

$$
\left[R_{p_{j}}^{2} S_{x}^{2}-R_{i}^{2} S_{x}^{2}\right] \leq 0
$$

or,

$$
R_{p_{j}} \leq \pm R_{i},(j=1,2,3),(i=18,19, \cdots, 26)
$$




\section{Empirical Example}

To judge the performances of the proposed and the existing estimators of population mean and to verify the conditions under which proposed estimators performs better than the existing estimators, we have considered the population given by Kadilar and Cingi [14]. The numerical values of the constants, biases and the mean squared error of the proposed and the existing estimators have been calculated for this data. The population parameters for the above population are as follows:

$$
\begin{aligned}
& N=106, \quad n=40, \quad \bar{Y}=2212.59, \quad \bar{X}=27421.70 \\
& \rho=0.860, \quad \rho=0.860, \quad C_{y}=5.22, \quad S_{x}=57460.61 \\
& C_{x}=2.10, \quad \beta_{1}=2.122, \quad \beta_{2}=34.572, \quad M_{d}=7297.50 \\
& Q D=12156.25, \quad G=40201.69, \quad D=35634.99, \quad S_{p w}=35298.81
\end{aligned}
$$

Table 2 represents the numerical values of constants, biases and the mean squared errors of proposed and other existing estimators of population mean using auxiliary variable for the above data.

\section{Results}

Form Table 2, we see that the proposed estimators are having lesser biases and mean squared errors as compared to all existing estimators. So the proposed es-

\begin{tabular}{|c|c|c|c|c|c|c|c|}
\hline Estimator & Constant & Bias & Mean Squared error & Estimator & Constant & Bias & Mean Squared error \\
\hline$t_{0}$ & Nil & 0 & 2077627.25 & $t_{15}$ & 0.0767 & 136.64 & 857402.20 \\
\hline$t_{r}$ & 0.0807 & 171.32 & 984589.70 & $t_{16}$ & 0.0801 & 148.10 & 884526.80 \\
\hline$t_{1}$ & 0.0807 & 151.20 & 889617.50 & $t_{17}$ & 0.0742 & 128.08 & 838466.80 \\
\hline$t_{2}$ & 0.0807 & 151.18 & 889566.40 & $t_{18}$ & 0.0327 & 24.87 & 610126.10 \\
\hline$t_{3}$ & 0.0806 & 150.82 & 888775.70 & $t_{19}$ & 0.0475 & 52.34 & 670914.00 \\
\hline$t_{4}$ & 0.0807 & 151.20 & 889616.00 & $t_{20}$ & 0.0297 & 20.59 & 600579.70 \\
\hline$t_{5}$ & 0.0806 & 151.02 & 889215.30 & $t_{21}$ & 0.0320 & 23.85 & 607875.10 \\
\hline$t_{6}$ & 0.0807 & 151.19 & 889596.60 & $t_{22}$ & 0.0498 & 57.60 & 682552.70 \\
\hline$t_{7}$ & 0.0807 & 151.20 & 889607.50 & $t_{23}$ & 0.0351 & 28.59 & 618381.50 \\
\hline$t_{8}$ & 0.0807 & 151.17 & 889557.80 & $t_{24}$ & 0.0322 & 24.12 & 608480.30 \\
\hline$t_{9}$ & 0.0867 & 151.20 & 889616.90 & $t_{25}$ & 0.0500 & 58.02 & 683478.00 \\
\hline$t_{10}$ & 0.0806 & 150.76 & 888634.40 & $t_{26}$ & 0.0353 & 28.90 & 619061.50 \\
\hline$t_{11}$ & 0.0807 & 151.14 & 889492.50 & $t_{p_{1}}$ & 0.00245 & 0.062 & 565007.83 \\
\hline$t_{12}$ & 0.0807 & 151.13 & 889452.90 & $t_{p_{2}}$ & 0.00262 & 0.064 & 565132.91 \\
\hline$t_{13}$ & 0.0637 & 94.32 & 763783.60 & $t_{p_{3}}$ & 0.00273 & 0.067 & 565334.48 \\
\hline$t_{14}$ & 0.0715 & 119.04 & 818477.40 & & & & \\
\hline
\end{tabular}
timators are more efficient than the other estimators for estimating population mean. Our purpose to search for the estimator with higher efficiency is achieved.

Table 2. Constants, Biases and MSE of Proposed and other estimators. 
Further it is to be mentioned that among the proposed estimators, $t_{p_{1}}$ is the best as it has smallest bias and the mean squared error.

\section{Conclusion}

This paper deals with the estimation of population mean of the study variable using auxiliary variable in the form of a special parameter along with some non-traditional measures of dispersion of auxiliary variable used by Abid et al. [1]. The expressions for the biases and mean squared errors of these proposed estimators have been derived up to the first order of approximation. A theoretical comparison of the proposed estimators has been made with the existing estimators of population mean under simple random sampling scheme. An empirical study is also carried out to judge the performances of the proposed and existing estimators of population mean. Through this numerical study, it has been found that the proposed estimators are more efficient than the other existing estimators. As proposed estimators are more efficient estimators for population mean, so they should be used for the improved estimation of population mean of study variable using auxiliary variable under simple random sampling scheme.

\section{Acknowledgements}

The authors are thankful to editor of Open Journal of Statistics and anonymous referees for critically examining the manuscript which helped in improving the earlier draft.

\section{References}

[1] Abid, M., Abbas, N., Sherwani, R.A.K. and Nazir, H.Z. (2016) Improved Ratio Estimators for the Population Mean Using Non-Conventional Measures of Dispersion. Pakistan Journal of Statistics and Operations Research, 12, 353-367.

[2] Cochran, W.G. (1940) Sampling Techniques. 3rd Edition, Wiley Eastern Limited, New Delhi.

[3] Yadav, S.K. (2012) Improved Exponential Ratio Cum Dual to Ratio Type Estimator of Population Mean. Econophysics Sociophysics and Other Multidisciplinary Science Journal, 2, 58-60.

[4] Yadav, S.K. and Kadilar, C. (2013) Improved Class of Ratio and Product Estimators, Applied Mathematics and Computation, 219, 10726-10731.

[5] Yadav, S.K. and Kadilar, C. (2013) Efficient Family of Exponential Estimator for Population Mean. Hacettepe Journal of Mathematics and Statistics, 42, 671-677.

[6] Yadav, S.K., Mishra, S.S. and Kumar, S. (2014) Optimal Search for Efficient Estimator of Finite Population Mean Using Auxiliary Information. American Journal of Operational Research, 4, 28-34.

[7] Yadav, S.K., Mishra, S.S. and Shukla, A.K. (2014) Improved Ratio Estimators for Population Mean Based on Median Using Linear Combination of Population Mean and Median of an Auxiliary Variable. American Journal of Operational Research, 4 , 21-27.

[8] Yadav, S.K., Mishra, S.S. and Shukla, A.K. (2015) Estimation Approach to Ratio of Two Inventory Population Means in Stratified Random Sampling. American Jour- 
nal of Operational Research, 5, 96-101.

[9] Yadav, S.K., Mishra, S.S., Shukla, A.K., Kumar, S. and Singh, R.S. (2016) Use of Non-Conventional Measures of Dispersion for Improved Estimation of Population Mean. American Journal of Operational Research, 6, 69-75.

[10] Yadav, S.K. and Mishra, S.S. (2015) Developing Improved Predictive Estimator for Finite Population Mean Using Auxiliary Information. Statistika, 95, 76-85.

[11] Misra, S. and Gupta, R.K. (2006) Estimation of Population Variance Using Ratio Type Estimator. Indian Journal of Mathematics and Mathematical Sciences, 2, 169176.

[12] Misra, S. and Gupta, R.K. (2008) Almost Unbiased Jacknifed Ratio Type Estimator of Population Variance. International Journal of Agricultural and Statistical Sciences (IJASS), 4, 345-350.

[13] Misra, S., Gupta, R.K. and Shukla, A.K. (2012) Generalized Class of Estimators for Estimation of Finite Population Variance. International Journal of Agricultural and Statistical Sciences, 8, 447-458.

[14] Kadilar, C. and Cingi, H. (2004) Ratio Estimators in Simple Random Sampling. Applied Mathematics and Computation, 151, 893-902.

[15] Kadilar, C. and Cingi, H. (2006) An Improvement in Estimating the Population Mean by Using the Correlation Coefficient. Hacettepe Journal of Mathematics and Statistics, 35, 103-109.

[16] Yan, Z. and Tian, B. (2010) Ratio Method to the Mean Estimation Using Coefficient of Skewness of Auxiliary Variable. In: Zhu, R., Zhang, Y., Liu, B. and Liu, C., Eds., Information Computing and Applications. ICICA 2010. Communications in Computer and Information Science, Vol. 106, Springer, Berlin, Heidelberg, 103-110. https://doi.org/10.1007/978-3-642-16339-5_14

[17] Subramani, J. and Kumarapandiyan, G. (2012) Estimation of Population Mean Using Co-Efficient of Variation and Median of an Auxiliary Variable. International Journal of Probability and Statistics, 1, 111-118.

[18] Jeelani, M.I., Maqbool, S. and Mir, S.A. (2013) Modified Ratio Estimators of Population Mean Using Linear Combination of Coefficient of Skewness and Quartile Deviation. International Journal of Modern Mathematical Sciences, 6, 174-183.

[19] Subramani, J. (2013) Generalized Modified Ratio Estimator for Estimation of Finite Population Mean. Journal of Modern Applied Statistical Methods, 12, 121-155. 


\section{Notations}

The following given by Abid [1] have been used in this manuscript and are as,

$N$ - Size of the population,

$n$ - Size of the sample,

$Y$ - Study variable,

$X$ - Auxiliary variable,

$\bar{Y}, \bar{X}$ - Population means,

$\bar{y}, \bar{x}$ - Sample means,

$S_{y}, S_{x}$ - Population Standard Deviations,

$S_{y x}$ - Population Covariance between $Y$ and $X$,

$C_{y}, C_{x}$ - Coefficients of Variation,

$M_{d}$ - Median of the auxiliary variable,

$\rho$ - Correlation coefficient between $X$ and $Y$,

$b=\frac{s_{y x}}{s_{x}^{2}}-$ Regression coefficient of $y$ on $x$,

$\beta_{1}=\frac{N \sum_{i=1}^{N}\left(X_{i}-\bar{X}\right)^{3}}{(N-1)(N-2) S_{x}^{3}}-$ Coefficient of Skewness of auxiliary variable,

$\beta_{2}=\frac{N(N+1) \sum_{i=1}^{N}\left(X_{i}-\bar{X}\right)^{4}}{(N-1)(N-2)(N-3) S_{x}^{4}}-\frac{3(N-1)^{2}}{(N-2)(N-3)}-$ Coefficient of Kurtosis of auxiliary variable,

$Q D=\frac{Q_{3}-Q_{1}}{2}-$ Quartile Deviation,

$G=\frac{4}{N-1} \sum_{i=1}^{N}\left(\frac{2 i-N-1}{2 N}\right) X_{i}$ - Gini'sMean Difference,

$D=\frac{2 \sqrt{\pi}}{N(N-1)} \sum_{i=1}^{N}\left(i-\frac{N+1}{2}\right) X_{i}$ - Downton's Parameters,

$S_{p w}=\frac{\sqrt{\pi}}{N^{2}} \sum_{i=1}^{N}(2 i-N-1) X_{i}$ - Probability Weighted Moments,

$B($.$) - Bias of the estimator,$

$V($.$) - Variance of the estimator,$

$M S E($.$) - Mean squared error of the estimator,$

$\operatorname{PRE}\left(t_{e}, t_{p}\right)=\frac{\operatorname{MSE}\left(t_{e}\right)}{\operatorname{MSE}\left(t_{p}\right)} * 100$ - Percentage relative efficiency of the estimator

$t_{p}$ over $t_{e}$. 
Submit or recommend next manuscript to SCIRP and we will provide best service for you:

Accepting pre-submission inquiries through Email, Facebook, LinkedIn, Twitter, etc. A wide selection of journals (inclusive of 9 subjects, more than 200 journals)

Providing 24-hour high-quality service

User-friendly online submission system

Fair and swift peer-review system

Efficient typesetting and proofreading procedure

Display of the result of downloads and visits, as well as the number of cited articles Maximum dissemination of your research work

Submit your manuscript at: http://papersubmission.scirp.org/

Or contact ojs@scirp.org 